\title{
TrkB Isoforms Differentially Affect AICD Production through Their Intracellular Functional Domains
}

\author{
Sara Ansaloni, Brian P. Leung, Neeraj P. Sebastian, Rohini Samudralwar, \\ Mariana Gadaleta, and Aleister J. Saunders \\ Department of Biology, Drexel University, Philadelphia, PA 19027, USA
}

Correspondence should be addressed to Aleister J. Saunders, aleister.saunders@drexel.edu

Received 2 September 2010; Revised 12 November 2010; Accepted 16 November 2010

Academic Editor: E. A. Rogaeva

Copyright (C) 2011 Sara Ansaloni et al. This is an open access article distributed under the Creative Commons Attribution License, which permits unrestricted use, distribution, and reproduction in any medium, provided the original work is properly cited.

\begin{abstract}
We report that NTRK2, the gene encoding for the TrkB receptor, can regulate APP metabolism, specifically AICD levels. Using the human neuroblastoma cell line SH-SY5Y, we characterized the effect of three TrkB isoforms (FL, SHC, T) on APP metabolism by knockdown and overexpression. We found that TrkB FL increases AICD-mediated transcription and APP levels while it decreases sAPP levels. These effects were mainly mediated by the tyrosine kinase activity of the receptor and partially by the PLC- $\gamma$ - and SHCbinding sites. The TrkB T truncated isoform did not have significant effects on APP metabolism when transfected by itself, while the TrkB SHC decreased AICD-mediated transcription. TrkB T abolished TrkB FL effects on APP metabolism when cotransfected with it while TrkB SHC cotransfected with TrkB FL still showed increased APP levels. In conclusion, we demonstrated that TrkB isoforms have differential effects on APP metabolism.
\end{abstract}

\section{Introduction}

Alzheimer's Disease (AD) is a neurodegenerative disorder that will affect 15 million people in USA alone in the next ten years $[1,2]$. The most common form of the disease is the late onset form (LOAD) that affects people older than 65. LOAD is caused by a complex interaction of risk factors including age, genetics, and environmental factors, such as level of education, diet, and physical activity [3-7].

The accumulation of $\mathrm{A} \beta$, a neurotoxic product of amyloid precursor protein (APP) cleavage, is central to $\mathrm{AD}$ pathogenesis [8-10]. This accumulation causes synaptic dysfunction and eventually neuronal death $[9,10]$. Therefore proteins that affect APP metabolism and synaptic function are likely to be important in $\mathrm{AD}$ pathogenesis.

TrkB is a member of the tyrosine kinase receptor family (Trk). TrkB specifically binds Brain-Derived Neurotrophic Factor (BDNF). The neurotrophin receptors (TrkA, TrkB, and $\operatorname{TrkC}$ ) are important in neuronal development and synaptic function $[11,12]$. Levels of TrkA, TrkB, and TrkC, but not $\mathrm{p} 75^{\mathrm{NTR}}$, are downregulated in $\mathrm{AD}$ brain samples [13]. Trk downregulation has been proposed as a biomarker of $\mathrm{AD}$ progression since Trk mRNA levels correlate with the degree of cognitive impairment [13].

Further evidence for a role of $\operatorname{TrkB}$ in $\mathrm{AD}$ is the fact that TrkB can modulate APP levels and proteolysis. Expression of the longest TrkB isoform, full-length TrkB (TrkB FL), can increase APP promoter transcription and promotes accumulation of sAPP- $\alpha$ [14-17]. Conversely, A $\beta$ has been found to reduce TrkB FL/BDNF levels and to impair TrkB-mediated signaling [18-21]. These results suggest a dynamic interaction between TrkB/BDNF signaling and APP metabolism.

Interestingly, knockdown of another splice variant of TrkB, truncated TrkB (TrkB T) in a mouse model of Down syndrome rescued neuronal death [22]. Conversely, mice overexpressing $\operatorname{TrkB} \mathrm{T}$ display synaptic dysfunction and long-term potentiation defects [23].

The gene encoding TrkB, NTRK2, is located on chromosome 9 , specifically $9 \mathrm{q} 22$. This region has been genetically linked to $\mathrm{AD}[24,25]$. Despite the experimental evidence functionally linking TrkB signaling to APP metabolism and synaptic function, case-control and genomewide association studies of NTRK2 single nucleotide polymorphisms (SNPs) 
found no significant association with $\mathrm{AD}$ [25-30]. One family-based study did observe genetic association of NTRK2 haplotypes with AD [26].

Three major splice variants of $\operatorname{TrkB}$ are expressed in neurons, TrkB FL, TrkB SHC, and TrkB T. We hypothesized that these different TrkB isoforms differentially affect APP metabolism and could play a role in the pathogenesis of AD. The aim of this work was to test this hypothesis.

The three TrkB splicing isoforms we investigated share an extracellular BDNF binding domain and differ in their cytoplasmic-domain (Figure 1) [31]. Two splice variants encode full-length receptors, TrkB full-length (FL), that contain a tyrosine kinase domain, an SHC-binding domain and a PLC- $\gamma$-binding domain in the intracellular portion [32, 33]. Two isoforms encode shorter receptors, TrkB SHC, that contain only an SHC-binding domain, and the remaining isoform is a truncated receptor, $\operatorname{TrkB} \mathrm{T}$, that does not have any known intracellular functional domain (Figure 1). Recently, isoforms differing in the extracellular domain have also been identified [34].

We used a previously described cell-based functional screen [35] to identify putative APP metabolism regulators. We found that NTRK2 knockdown altered both AICDmediated luciferase activity and APP full-length levels. To characterize the role of TrkB FL and truncated isoforms we knocked down and overexpressed the isoforms in an $\mathrm{SH}$ SY5Y neuroblastoma cell line overexpressing APP as a fusion protein with the yeast transcription factor Gal-4 [35]. We then measured APP FL levels and proteolytic products using Western blots and luciferase assays.

Our results demonstrate for the first time that TrkB isoforms differentially affect APP metabolism. Specifically, overexpression of TrkB FL increases AICD-Gal4-mediated luciferase activity. While overexpression of $\operatorname{TrkB} \mathrm{T}$ does not alter the luciferase activity and TrkB SHC decreases the luciferase activity compared to control. We determined that the tyrosine kinase and PLC- $\gamma$ functional domains contribute to the observed TrkB FL-mediated effects. We also found that the SHC-binding site contributed to the observed TrkB-SHC-mediated effects. BDNF stimulation of the exogenously expressed $\operatorname{TrkB}$ receptors amplified the APP metabolism effects and cotransfection of the TrkBtruncated isoforms with TrkB FL altered the effects on APP metabolism.

\section{Materials and Methods}

2.1. Constructs and Site-Directed-Mutagenesis. Four shRNAcontaining plasmids specific for NTRK2 were obtained from the pSM2 retroviral Library of the Drexel RNAi Resource Center purchased from Open Biosystems. The constructs ID numbers are NTRK2.1: 1920; NTRK2.2: 2295; NTRK2.3: 29734; NTRK2.4: 30795. We also used APP (ID 39147) and luciferase targeting shRNA (RHS1705) as positive controls and a scrambled shRNA sequence (nonsilencing, NS, RHS1707) as a negative control. The TrkB full-length and truncated GFP fusion constructs and the GFP-F control overexpression plasmid were kindly donated

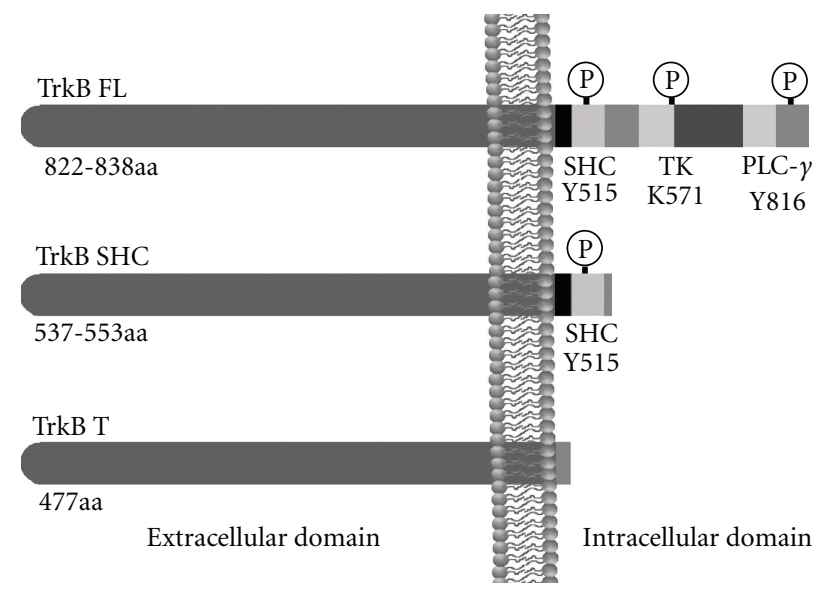

Figure 1: TrkB isoforms structure and functional domains. The extracellular portion of the receptor is conserved. The intracellular domain differs among the isoforms. TrkB FL contains a tyrosine kinase domain, a SHC-binding domain, and a PLC- $\gamma$-binding domain. TrkB SHC contains a SHC-binding domain and TrkB T does not have any functional domain.

by Dr. Eero Castren (University of Helsinki, Finland) and were previously described [36, 37]. Site-directed mutagenesis (Stratagene, Quikchange mutagenesis kit) was utilized to generate point mutants on the $\operatorname{TrkB}$ full-length receptor functional domains. Mutagenesis was carried out according to manufacturer's instructions and the primers employed are reported in Table 1 (the bolded sequences represent the mutations/insertion). The mutant amino acid indicated refers to the amino acidic sequence of TrkB. Therefore TrkB FL K571M indicates the tyrosine kinase dead receptor since it is mutated on the ATP-binding site; TrkB FL Y515F indicates the receptor mutated on the SHC-binding site; TrkB FL Y816F indicates the receptor mutated on the PLC- $\gamma$-binding site [38]. Note that in some literature the TrkB mutants are referred to with the numbering of the amino acidic sequence of TrkA, the NGF receptor, that has functional sites in common with TrkB and therefore are referred to as $\mathrm{K} 560 \mathrm{M}$, Y490F and Y785F respectively $[32,33]$. TrkB SHC indicates the other human truncated isoform (isoform $\mathrm{d}$ and e, NCBI Gene NM_001018064.2 and NM_001018066.2). TrkB SHC was obtained by insertion of the exon 19 followed by a STOP codon after the SHC-binding site on the TrkB FL constructs. After obtaining the TrkB SHC isoform by insertion, the SHCbinding site was mutated on that isoforms using the same primer sequence employed for the TrkB FL mutant on the SHC-binding site (TrkB Y515F).

Successful mutations were identified by sequencing. One clone per construct was transformed in E. coli (DH5- $\alpha$ competent cells, InVitrogen). Transformed bacteria were selected on $100 \mu \mathrm{g} / \mathrm{mL}$ ampicillin LB-agar plates and liquid cultures were grown overnight at $37^{\circ} \mathrm{C}$. Bacterial cultures were miniprepped (MiniPrep Kit, Quiagen) and used for transfection after DNA quantification.

2.2. Cell Culture and Transfection. SH-SY5Y cells stably transfected with UAS-Luciferase and APP-Gal4 described 


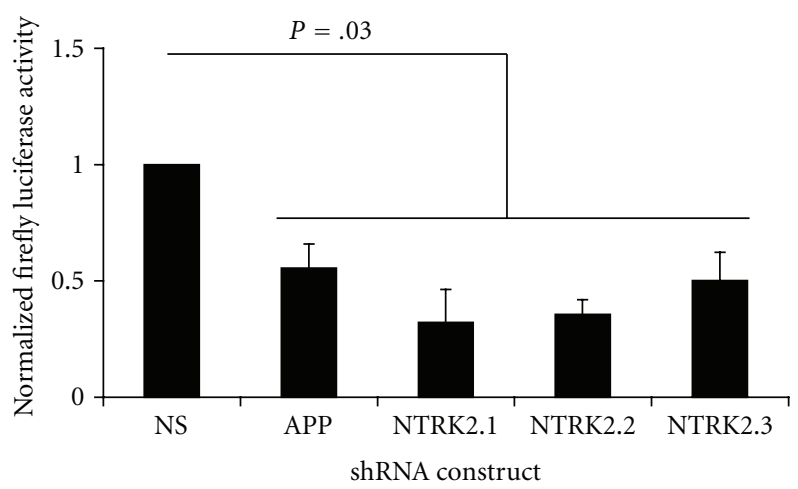

(a)

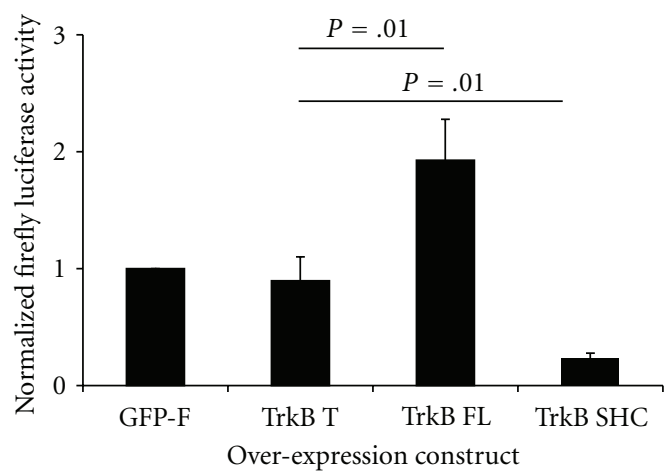

(b)

FIgURE 2: AICD-mediated Luciferase activity in SH-SY5Y-APP-Gal4 cells with TrkB knockdown or TrkB overexpression. (a) shRNAmediated NTRK2 knock-down. Luciferase activity is decreased by NTRK2 knockdown compared to nonsilencing control $(P=.03)$. There is no significant difference between luciferase activity in NTRK2 knockdown and APP knockdown cells. shRNA constructs: negative control, NS, scrambled shRNA sequence; positive control, APP, shRNA targeting APP; experimental shRNA, NTRK2.1-3, shRNA constructs targeting all TrkB isoforms. The experiment was repeated four times independently and each time with six replicates. (b) TrkB isoform overexpression. Luciferase activity was not altered by TrkB T compared to GFP control. Luciferase activity was increased by TrkB FL $(P=.01)$ and decreased by TrkB SHC $(P=.01)$ compared to a GFP-control vector. The experiment was repeated 3 to 6 times independently with 6 replicates each time. The $y$-axis represents the arbitrary light units of firefly luciferase normalized to cell number (a) or transfection efficiency (b). Error bars $=$ SEM. Statistical analysis: ANOVA and Students $t$-test with Bonferroni correction where applicable.

TABle 1: Primer sequences used to obtain the point mutations and the sequence insertion on the TrkB FL receptor. The mutated bases are represented in bold.

\begin{tabular}{ll}
\hline Primers & Sequence $5^{\prime}$ to $3^{\prime}$ \\
\hline TrkB.Y490F.F & GTCATTGAAAACCCCCAGTTCTTCGGTATCACCAACAG \\
TrkB.Y490F.R & CTGTTGGTGATACCGAAGAACTGGGGGTTTTCAATGAC \\
TrkB.Y816F.F & GCGTCGCCCGTCTTCCTGGACATCCTAG \\
TrkB.Y816F.R & CGCAGCGGGCAGAAGGACCTGTAGGATC \\
TrkB.Shc.F & CTCAAGCCGGACACATGGCCCAGAGGTTCCCCCAAGACCGCCTGATAGTAATTTGTTCAGCACATC \\
TrkB.Shc.R & GATGTGCTGAACAAATTACTATCAGGCGGTCTTGGGGGAACCTCTGGGCCATGTGTCCGGCTTGAG \\
TrkB K560M.F & GGTGGCCGTGATGACGCTGAAGG \\
TrkB K560M.R & CCTTCAGCGTCATCACGGCCACC \\
TrkB.SHC & GTCATTGAAAACCCCCAGTTCTTCGGTATCACCAACAG \\
TrkB.SHC & CTGTTGGTGATACCGAAGAACTGGGGGTTTTCAATGAC \\
\hline
\end{tabular}

before [35] were maintained in DMEM (Gibco) supplemented with $10 \%$ FBS, penicillin streptomycin and $200 \mu \mathrm{g} / \mathrm{mL}$ G418 (Gibco).

To assess the effects of TrkB knockdown or overexpression on AICD-Gal4 mediated luciferase we used the following transfection protocol previously described [35]. Briefly, one day before transfection cells were plated in 96wells plates at approximately $40-50 \%$ confluency. The day of transfection media was removed from the cells and replaced with transfection media: $100 \mu \mathrm{L}$ of serum free DMEM media containing $2 \mu \mathrm{g} /$ well Arrest-In (Open Biosystems) and $0.2 \mu \mathrm{g} /$ well plasmid DNA. The pSM2 plasmids referred to as NTRK2.1 through 4 were transfected in the cells. Cells were also transfected with shRNA targeting APP, luciferase and a control shRNA that contains a scrambled sequence that does not target any human gene. In addition a mock transfection, containing only Arrest-In was performed to control for selection effectiveness. 6 replicate wells per
shRNA constructs and mock control transfection were set up for each independent experiment. The transfection media was left on the cells for 8 hours and then replaced with complete media. 48 hours after transfection, transfected cells were selected with $4 \mu \mathrm{g} / \mathrm{mL}$ puromycin (Sigma) in $10 \%$ FBS DMEM with $200 \mu \mathrm{g} / \mathrm{mL}$ G418. Puromycin selection was used to preserve transiently transfected cells. The media was changed every 48 hours and cell death was monitored and compared to the mock-transfected control. Once all the cells in the mock control wells were dead, surviving cells in the shRNA transfected wells were split and transferred to another 96-well plate and a 24-well plate. Cell lysates were collected from 60-80\% confluent 96-wells 11-13 days after transfection in $100 \mu \mathrm{L}$ Glo Lysis Buffer per well (Promega). Lysates were used immediately after collection or frozen prior to performing Steady Glo luciferase assays (Promega). The luciferase assays were performed as per manufacturer's instructions. shRNA-mediated knockdown effectiveness was 


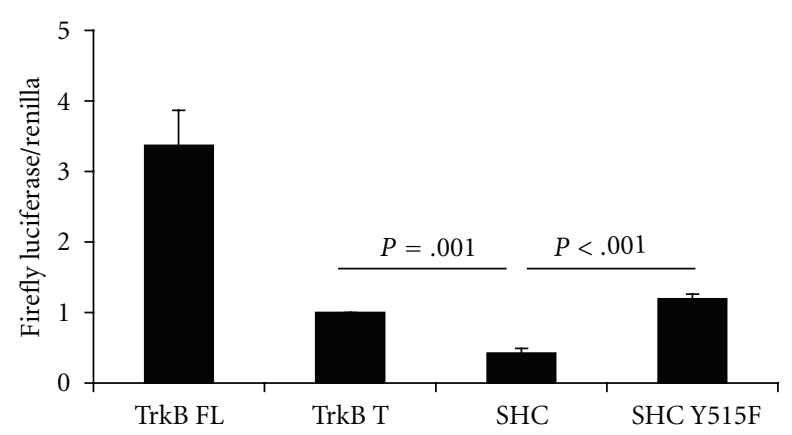

(a)

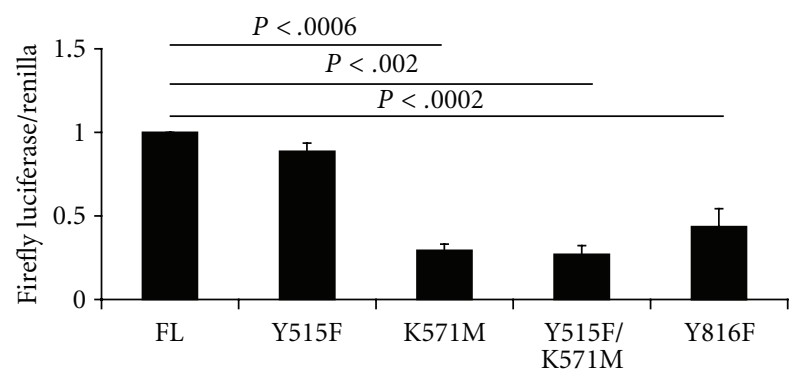

(b)

FIgUre 3: Mutation of TrkB FL intracellular functional domains changes the effect on AICD-mediated luciferase activity. (a) The TrkB SHC isoform significantly decreases luciferase activity when compared to TrkB T $(P=.001)$. The TrkB SHC Y515F mutant, unable to bind SHC, increases luciferase activity compared to TrkB SHC $(P<.001)$ and does not significantly alter luciferase activity compared to TrkB T $(P=.1)$. (b) TrkB Y515F (no SHC-binding) does not significantly alter luciferase activity compared to TrkB FL. TrkB K571M with an inactive tyrosine kinase domain significantly decreases luciferase activity compared to TrkB FL $(P=.0006)$; TrkB Y515F/K571M significantly decreases luciferase activity compared to $\operatorname{TrkB} \mathrm{FL}(P=.002)$ but not to a greater extent of TrkB K571M. TrkB Y816F that does not bind PLC- $y$ also significantly decreases luciferase activity compared to $\operatorname{TrkB}$ FL $(P=.0002) .4-8$ independent experiments with 6 replicates each, error bars SEM. 4 6 independent experiments with 6 replicates each, error bars SEM. Statistical analysis: Student's $t$-test with Bonferroni correction.

monitored by comparing the luciferase signal of the nonsilencing control shRNA with the APP targeting shRNA. After assessing successful knock-down, luciferase data for the experimental shRNA targeting NTRK2 was collected and analyzed. In parallel, 24-well plates and 12-well plates were seeded with the same cells that had been assayed for luciferase signal and collected for Western Blot analysis.

The same transfection procedure was followed for the overexpression experiments, but lysates were collected 48 hours after transfection and transfection efficiency was monitored by fluorescence microscopy, no antibiotic selection was performed in this case.

2.3. Western Blotting Procedures. Conditioned media was collected from the cells (48 hours after transfection) in eppendorf tubes and centrifuged at $14,000 \mathrm{rpm}$ for 10 minutes at $4^{\circ} \mathrm{C}$ (Beckman Coulter, Microfuge 22R). The resulting supernatant was collected and $142 \mu \mathrm{L}$ were mixed with $33 \mu \mathrm{L}$ of $4 \mathrm{X}$ Reducing loading buffer (InVitrogen) supplemented with $0.4 \% \beta$-mercaptoethanol (Sigma). These samples were heated at $70^{\circ} \mathrm{C}$ for 10 minutes. The remaining conditioned media was stored at $-20^{\circ} \mathrm{C}$ for later Western Blot analysis.

Whole cell lysates were collected (48 hours after transfection) by lysing the cells with ice-cold radio immunoprecipitation (RIPA) buffer $(150 \mathrm{mM} \mathrm{NaCl}, 1 \% \mathrm{NP} 40$, $0.5 \%$ DOC, $1 \%$ SDS, $50 \mathrm{mM}$ Tris, $\mathrm{pH} 8.0$ ) supplemented with Halt cocktail of protease and phosphatase inhibitors (ThermoScientific). Cell lysates were sonicated in an icecold water bath sonicator for 6 minutes then centrifuged 20 minutes at $4^{\circ} \mathrm{C}$ at $14,000 \mathrm{rpm}$. The resulting supernatants were collected and protein concentration measured with a BCA protein concentration kit (Pierce) according to manufacturer's instructions.

Western blot samples were prepared at a final concentration of $1-2 \mu \mathrm{g} / \mu \mathrm{L}$ in $4 \mathrm{X}$ reducing loading buffer (InVitrogen) and heated at $70^{\circ} \mathrm{C}$ for 10 minutes. $15-25 \mu \mathrm{g}$ of total protein/well were separated on 4-12\% Tris-Glycine midi gels (InVitrogen) in MES-SDS running buffer (InVitrogen) and run at 190 mVolts for 45 minutes. The separated proteins were transferred to PVDF FL membranes (Millipore) in a Semi-Dry transfer apparatus (AA Hoefer TE77X) for 3 hours at 125 milli Amp per gel.

Membranes were blocked one hour at room temperature using Licor blocking buffer then probed overnight with primary antibodies diluted in Licor blocking buffer at 4 or $25^{\circ} \mathrm{C}$. Membranes were then washed for 5 minutes 4 times with $0.1 \%$ Tween (Sigma) in PBS. One last wash was performed with PBS to rinse off the detergent. After washing, membranes were incubated in the dark with the appropriate secondary antibody IRDye (Licor) diluted in Licor blocking buffer for one hour. Again membranes were washed as above and finally rinsed with PBS. Membranes were scanned on an Odyssey InfraRed scanner (Licor) at appropriate intensities and images acquired. Band intensities were quantified with the provided in-built software (Licor) and always normalized to the actin loading control. When conditioned media was analyzed the signals were normalized to the protein concentration of the corresponding lysates.

2.4. Antibodies. Detection of TrkB-GFP tagged constructs utilized mouse anti-GFP antibody $(1: 1000$, Living Colors, Clontech); detection of APP full-length and C-terminal fragments utilized A8717 rabbit antibody $(1: 2000, \mathrm{Rb}$, Sigma); detection of sAPP $22 \mathrm{C} 11$ utilized mouse antibody $(1: 1000$, Millipore); detection of sAPP- $\alpha$ 6E10 utilized mouse antibody (1:1000, Covance); detection of actin A5441 utilized mouse antibody $(1: 15,000$, Sigma). The secondary antibodies: IRDye700 anti-mouse antibody $(1: 15,000)$ and IRDye800 anti-rabbit antibody $(1: 15,000)$ were obtained from by Licor.

\section{Results}

3.1. NTRK2 Knockdown Decreases AICD-Mediated Luciferase Activity. We applied our functional screening method [35] 


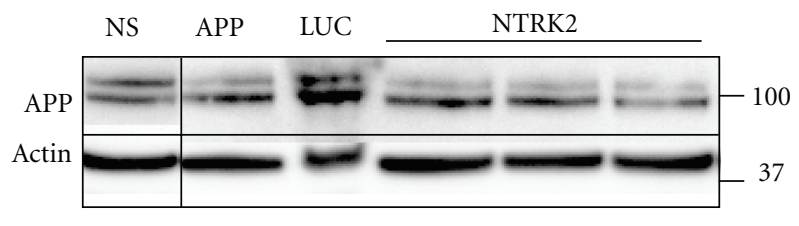

(a)

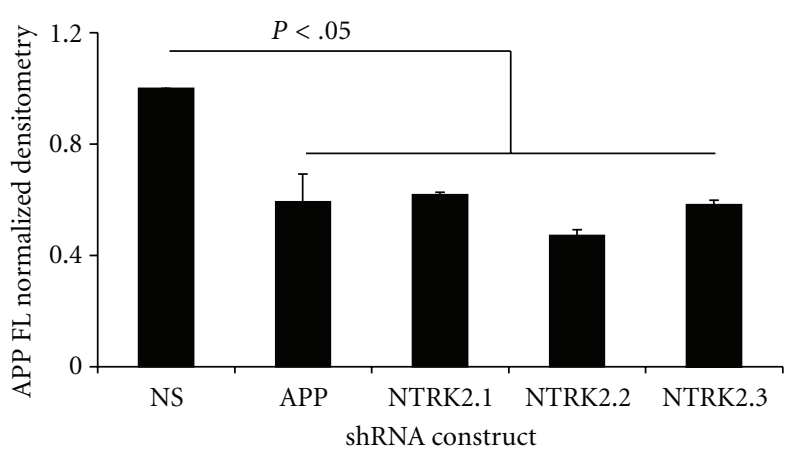

(b)

FIGURE 4: NTRK2 knockdown decreases APP full-length levels. (a) Representative Western Blot of APP full-length and actin as a loading control in lysates from cells transfected with shRNA constructs targeting APP, luciferase (LUC), NTRK2 and a negative, nonsilencing (NS), control. (b) Average quantification of APP full-length levels from four independent Western Blot analysis conducted on independent transfections. Within each transfection APP FL densitometry was normalized to the APP FL densitometry of the cell lysate obtained from cells transfected with CTRL shRNA. A statistically significant difference was observed between CTRL and the APP or NTRK2 shRNA targeting constructs $(P<.05)$. The experiment was repeated four times independently with three replicates each, error bars indicate SEM. Statistical analysis: ANOVA.

to all the genes in the linkage region on chromosome 9 that displays a high likelihood of disease score for AD [39]. This screening is conducted in SH-SY5Y cells stably transfected with a luciferase reporter driven by the yeast UAS promoter and APP fused to Gal4. When APP is cleaved by the secretases, the AICD-Gal4 domain is released and can activate the transcription of the luciferase reporter. Variations in AICDmediated luciferase activity are measured. Since changes in AICD-mediated luciferase activity can occur through a variety of mechanisms affecting APP, this is an effective and general way of identifying regulators of APP metabolism [35]. We targeted NTRK2 with 4 different shRNA constructs (see supplementary Figures 1 and 2 in the supplementary material available online at doi: 10.4061/2011/729382). Three shRNAs targeted all the TrkB isoforms (NTRK2.13) and one (NTRK2.4) targeted all the isoforms except the TrkB T. We also transfected a nonsilencing scrambled shRNA (NS) that does not target any human gene as a negative control and a shRNA targeting APP as a positive control. Of the four transfected constructs NTRK2.1-3 decreased AICDmediated luciferase to the same extent of the APP targeting shRNA compared to the NS shRNA (Figure 2(a)). The fourth construct, NTRK2.4 targeting all TrkB isoforms except TrkB $\mathrm{T}$, consistently caused cell death (data not shown). This result suggests that NTRK2 can affect APP metabolism and that the isoforms have different roles since downregulation of all the isoforms except TrkB T was lethal. Therefore we investigated the effect of the single isoforms in the same experimental model.

We transiently transfected individual TrkB isoform overexpression constructs in the cells and measured AICDmediated luciferase activity. We found that there was no difference in AICD-mediated luciferase activity between $\operatorname{TrkB} \mathrm{T}$ and the GFP-control, while TrkB FL significantly increased luciferase activity $(P=.01)$ and TrkB SHC significantly decreased it $(P=.01)$ (Figure 2(b)). These results demonstrate that TrkB isoforms have different effects on APP metabolism. Moreover, we show that there is a difference between the isoforms TrkB SHC and TrkB T, even though both isoforms lack the tyrosine kinase domain.

3.2. The Tyrosine Kinase and PLC- $\gamma$-Binding Domains of TrkB $F L$ and the SHC-Binding Domain on TrkB SHC Determine the Effect on AICD-Mediated Luciferase Activity. TrkB T did not alter AICD-mediated luciferase activity compared to the GFP-F control, while TrkB SHC decreased it and TrkB FL increased it. We hypothesized that the intracellular domains of the TrkB SHC and TrkB FL are responsible for the effects observed. To determine which domain was responsible for this effect, we mutated each cytoplasmic functional domain individually.

We generated a mutant of the TrkB SHC isoform that cannot bind SHC (Y515F). We transfected this mutant and the other TrkB wild-type isoforms, into SH-SY5Y-APP-Gal4 cells and measured AICD-mediated luciferase activity. We observed that TrkB SHC Y515F (SHC-binding site mutant) does not significantly alter luciferase activity compared to TrkB $\mathrm{T}$ but significantly increased it compared to the TrkB SHC wild-type isoform $(P<.001)$ (Figure 3(a)). Therefore, disrupting the SHC-binding site on the TrkB SHC isoform impairs its ability to decrease AICD-mediated luciferase activity.

We then generated mutants in the three functional sites of the TrkB FL. We mutated the SHC-binding site (Y515F) to generate a mutant that cannot bind SHC [33]. Then we mutated the ATP-binding site (K571M) to generate a TrkB FL tyrosine kinase inactive receptor K571M [32, 33]. Similarly, we disrupted the PLC- $\gamma$-binding site by introducing the mutation Y816F. We also generated a double mutant that is both tyrosine kinase inactive and does not bind SHC (TrkB Y515F/K571M). We then transfected these TrkB FL mutant constructs in SH-SY5Y-APP-Gal4 cells. We measured the AICD-mediated luciferase activity and compared it to $\operatorname{TrkB}$ FL wild-type (Figure 3(b)). 

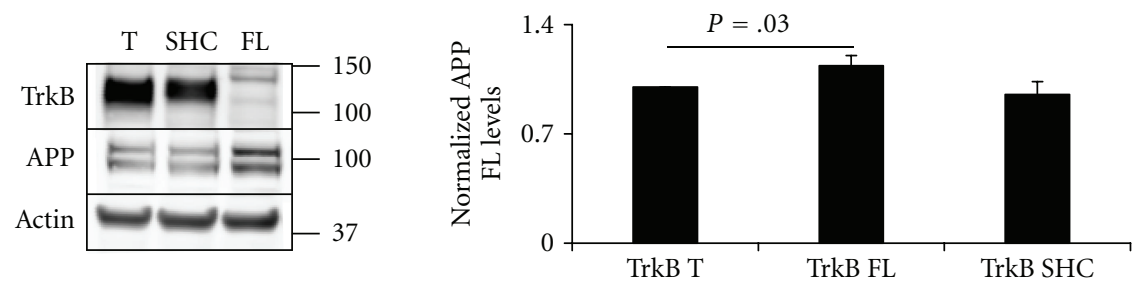

(a)
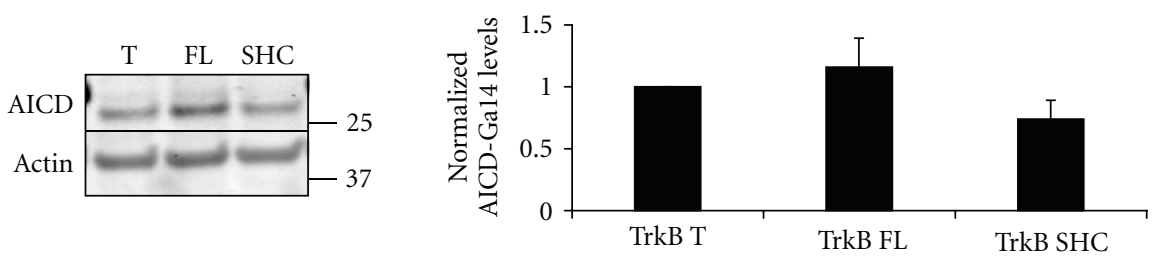

(b)
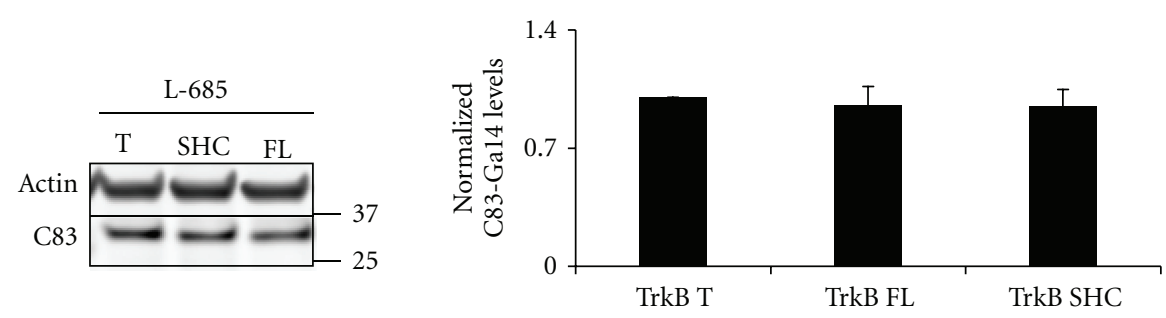

(c)

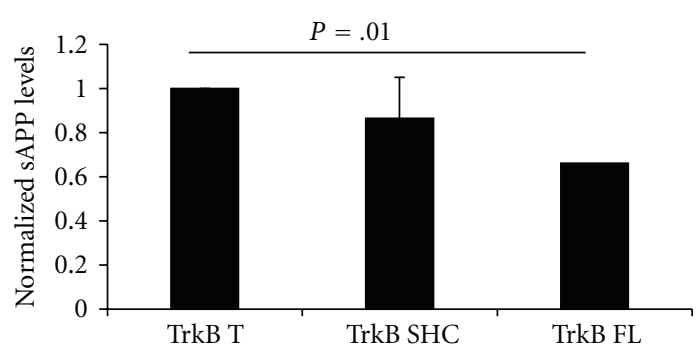

(d)

Figure 5: TrkB isoform overexpression affects APP FL levels and APP processing. (a) TrkB FL increases APP FL levels compared to TrkB $\mathrm{T}(P=.03)$ and TrkB SHC isoforms $(P=.008) .5$ independent experiments in duplicate, error bars indicate SEM. (b) TrkB FL increases AICD levels compared to TrkB T and TrkB SHC decreases AICD levels. (c) TrkB FL, TrkB T, and TrkB SHC do not alter C83 levels. Cells were treated with L-685,485 to aid in C83-Gal4 visulaization (d) sAPP levels in conditioned media. TrkB FL significantly decreases sAPP levels compared to TrkB T $(P=.003)$. The experiment was repeated four times independently in duplicate. Error bars indicate SEM. Statistical analysis: Students $t$-test with Bonferroni correction.

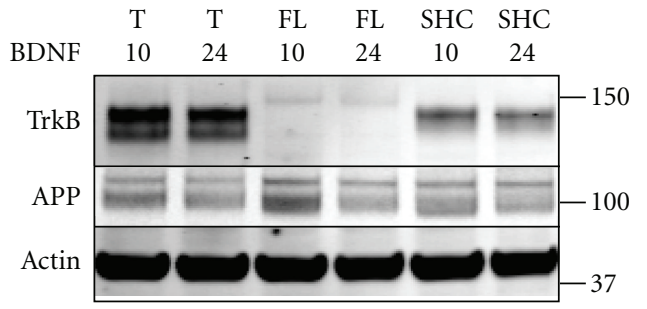

FIgURE 6: Short-term or long-term BDNF treatment of TrkB isoforms transfected cells. APP full-length (FL) levels are increased by a 10 minutes BDNF treatment of TrkB FL transfected cells while a 24 hours treatment shows lower APP FL levels. TrkB T and TrkB SHC transfected cells are not significantly affected by short term or long-term BDNF treatment.
The TrkB Y515F mutant (preventing SHC-binding) did not significantly alter AICD-mediated luciferase activity compared to TrkB FL (Figure 3(b)). The TrkB FL K571M (tyrosine kinase inactive) significantly decreased luciferase activity compared to TrkB FL $(P=.0006)$. TrkB FL Y816F, (preventing PLC- $y$-binding) also significantly decreased luciferase activity compared to $\operatorname{TrkB}$ FL $(P=.0002)$. The double mutant TrkB Y515F/K571M (preventing SHCbinding and tyrosine kinase inactive) significantly decreased luciferase compared to $\operatorname{TrkB}$ FL $(P=.002)$ but did not differ from the tyrosine kinase inactive TrkB K571M (Figure 2(a)).

In summary, TrkB FL overexpression increases AICDGal4 mediated luciferase activity two-fold compared to 


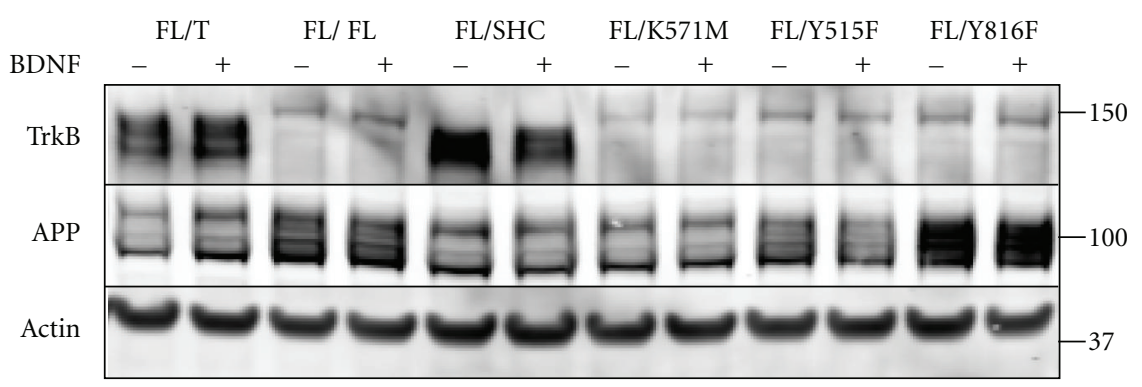

Figure 7: Cotransfection of TrkB FL with isoforms and mutants alters the TrkB FL effects on APP full-length. TrkB FL transfection increases APP FL levels compared to when TrkB FL is cotransfected with TrkB T, TrkB SHC or TrkB K571M. APP FL levels did not significantly differ between TrkB FL transfected cells and TrkB FL cotransfection with TrkB Y515F or TrkB Y816F. 24 hours BDNF treatment of cotransfected cells did not significantly alter APP FL levels compared to cotransfected but nontreated cells.

controls TrkB T (Figure 2(b)). The tyrosine kinase inactive mutant receptor, TrkB K571M, the PLC- $\gamma$-binding site mutant, and the TrkB SHC isoform mutated on the SHCbinding site also cause a $60-70 \%$ decrease in AICD-mediated luciferase activity compared to the TrkB FL (Figures 3(a) and 3(b)). The TrkB SHC wild-type isoform causes an AICDmediated luciferase decrease of about 90\% (Figure 2(b)).

3.3. NTRK2 Knockdown Decreases APP FL Levels. The effects we observe on AICD-mediated luciferase activity can occur through many different mechanisms: decreased APP transcription, increased APP degradation, decreased APP cleavage, destabilization of AICD, and trafficking that affects APP localization. Anything that decreases AICD levels will be reflected in a decrease in luciferase activity. The most immediate way of decreasing AICD levels is to decrease APP levels. To determine if NTRK2 knockdown decreased APP levels, we tested if APP levels were altered. We transfected the NTRK2 targeting shRNA, a CTRL shRNA, and an APP targeting shRNA as a positive control. As an additional control we used shRNA targeting the luciferase gene: this construct accounts for overexpression of shRNA that have to be processed by the endogenous RNAi machinery. We then measured APP protein levels by Western blot (Figure 4(a)). We found that knockdown of all the TrkB splice variants cause a significant decrease in APP FL levels $(P<.05)$ (Figure 4(b)) and we concluded that decreased APP levels might be at least partially responsible for the observed reduction in luciferase activity.

3.4. TrkB FL Overexpression Increases APP FL Levels and AICD Gal4 Levels. Based on the previous knockdown results, we then hypothesized that overexpression of TrkB FL causes increased AICD-mediated luciferase activity by increasing APP FL levels. We transfected the TrkB isoforms in the cells, performed Western blot analysis and quantified APP FL levels in cell lysates. Overexpression of TrkB FL significantly increased APP FL levels compared to TrkB T $(P=.03)$ and TrkB SHC $(P=.008)$ (Figure 5(a)). There was no difference in APP levels between TrkB T- and TrkB SHC-transfected cells (Figure 5(a)).

We then verified that AICD-Gal4 levels in TrkB FLtransfected cells correlated to the observed increase in luciferase activity. AICD-Gal4 is the intracellular domain of APP that is, generated by $\gamma$-secretase cleavage, translocates to the nucleus and activates transcription. We found that, as expected, TrkB FL displayed increased AICD-Gal4 levels compared to TrkB T, but this difference was not statistically significant. Compared to TrkB T, TrkB SHC overexpression resulted in a decrease in AICD-Gal4 levels, as expected, but this difference was not statistically significant (Figure 5(b)). Interestingly, we consistently observed TrkB FL lower levels compared to TrkB T and TrkB SHC in our Western blot analysis (Figure 5(a)).

3.5. TrkB FL Overexpression Decreases sAPP Levels without Altering C83 Levels. To assess changes in APP proteolysis we measured APP C-terminal fragments (CTFs) and sAPP levels upon TrkB transfection. CTFs include both C83 and C99. C83 and C99 are generated by the cleavage of APP by $\alpha$-secretase and $\beta$-secretase, respectively. In our cell line we measure C83-Gal4 and C99-Gal4 levels since APP overexpressed is a fusion protein with Gal4. These fragments are the precursors of AICD, that is, released in the cytoplasm by $\gamma$-secretase cleavage $[40,41]$. While C83 and C99 are membrane-bound fragments of APP, the soluble N-terminal fragment of APP, sAPP, generated by $\alpha / \beta$-secretase cleavage is released in the extracellular environment. In SH-SY5Y cells, the $\beta$-secretase cleavage occurs to a much lower extent than $\alpha$-secretase cleavage. Therefore, the majority of the luciferase signal observed is due to AICD-Gal4 generated from $\gamma$ secretase cleavage of C83-Gal4. If the AICD-Gal4 levels are increased, as measured by luciferase and Western blot, then the levels of its precursor C83-Gal4 should also be increased.

We then tested the hypothesis that C83-Gal4 and sAPP levels are increased by TrkB FL overexpression and decreased by TrkB SHC. To aid detection of C83-Gal4 we treated cells with the $\gamma$-secretase inhibitor L-685,485. We did not detect a difference in C83-Gal4 levels among the cells transfected with the different TrkB isoforms (Figure 5(c)). Surprisingly, TrkB FL decreased sAPP levels compared to TrkB T $(P=.01)$. TrkB SHC showed a nonsignificant difference in sAPP levels compared to TrkB T (Figure 5(d)).

3.6. BDNF Treatment of TrkB Isoforms Does Not Significantly Alter Their Effects on APP Metabolism. All three TrkB 
isoforms studied here are capable of binding BDNF [12]. Moreover, it has been previously shown that TrkB FL BDNFmediated intra-cellular signaling can alter APP metabolism [14-17]. We hypothesized that application of exogenous BDNF would stimulate the TrkB FL-mediated effects on APP FL and proteolytic products levels. We then tested this hypothesis applying BDNF to cells transfected with TrkB isoforms and measured the levels of APP FL by Western blot. We found that short term (10 minutes) BDNF application increases APP FL levels in cells transfected with the TrkB $\mathrm{T}$ or TrkB SHC isoforms and to a greater degree in cells that had been transfected with TrkB FL (Figure 6). Twentyfour hour BDNF treatment of TrkB FL transfected cells did not further increase APP FL levels compared to short-term BDNF treatment.

3.7. Cotransfection of the TrkB Isoforms Modulates TrkB FLMediated Effects on APP Metabolism. It has been previously shown that TrkB $\mathrm{T}$ has a dominant negative effect on TrkB FL [42]. We hypothesized that cotransfection of TrkB T with TrkB FL would eliminate the TrkB FL effects on APP metabolism observed when we transfect TrkB FL alone. Moreover we hypothesized that cotransfection of the TrkB SHC with TrkB FL would also have dominant negative effect on TrkB FL. Finally we hypothesized that cotransfection of TrkB FL with TrkB Y515F or TrkB Y816F would not significantly alter the effects seen on APP since they seem to be primarily mediated by the tyrosine kinase domain and not by the SHC-binding domain. For this reason we also hypothesized that cotransfection of TrkB FL with TrkB $\mathrm{K} 571 \mathrm{M}$ (TrkB FL/K571M) would have the same effect as the cotransfection of TrkB FL and TrkB T (TrkB FL/T).

Consistent with our hypothesis, TrkB FL/T cotransfection did not increase APP FL levels, nor did cotransfection of $\operatorname{TrkB} \mathrm{FL} / \mathrm{K} 571 \mathrm{M}$, the tyrosine kinase inactive mutant (Figure 7). Also, as expected, there was very little difference between the APP FL levels in cells transfected with TrkB FL/Y515F, TrkB FL/Y816F, and TrkB FL/FL. Surprisingly, cotransfection of TrkB FL/SHC increased APP FL levels compared to TrkB FL/T cotransfection but not compared to TrkB FL/FL (Figure 7).

We also hypothesized that BDNF treatment of the cotransfected cells would affect the transfected isoforms mediated effects on APP. Surprisingly BDNF treatment did not significantly alter these effects of the cotransfected TrkB receptors.

In summary, both truncated isoforms were able to decrease APP FL levels compared to TrkB FL/FL transfection; TrkB $\mathrm{T}$ to a greater extent than TrkB SHC. The tyrosine kinase inactive receptor decreased APP FL levels to the same extent of TrkB FL/T cotransfection while TrkB FL/Y515F and TrkB FL/Y816F cotransfection did not alter APP FL levels compared to TrkB FL/FL.

\section{Discussion}

We investigated the role of the $\operatorname{TrkB}$ isoforms on APP metabolism in SH-SY5Y cells overexpressing an APP-Gal4 fusion protein that can transactivate a luciferase reporter gene [35]. This system monitors changes in APP metabolism that are reflected in altered AICD-mediated transcription of the luciferase gene [35].

We found that knockdown of all TrkB isoforms in SHSY5Y-APP-Gal4 cells caused a decrease in AICD-mediated luciferase activity. This decrease is probably due to a decrease in APP levels observed in cells with NTRK2 knockdown. We hypothesize that decreased APP levels in this system are mainly due to increased APP degradation caused by altered trafficking in absence of TrkB. Transcriptional downregulation of APP might be partially responsible for the decreased signal observed in the Western Blot but that is only possible for the endogenous APP. Because the endogenous $A P P$ gene is under the physiologic transcriptional regulation, while the APP-Gal4 overexpressed is under CMV promoter regulation.

Concomitant knockdown of the TrkB FL and SHC isoforms lead to cell death, and this is consistent with the finding that TrkB $\mathrm{T}$ is one of the causes of neuronal death in a mouse model of trisomy 21 [43].

To discriminate between the effects of the different isoforms, we overexpressed one isoform at a time and measured the resulting AICD-mediated luciferase activity. As a control we employed a GFP expression vector (GFP-F) that includes a farnesylation sequence that targets GFP to the cell membrane. This is a better control for a membrane-bound receptor than a cytoplasmatic GFP. Interestingly, we observed isoform specific effects. TrkB FL increased luciferase activity while no difference was observed between TrkB T and GFPF control transfected cells. TrkB SHC induced a decrease in AICD-mediated luciferase activity. We hypothesize that the decrease in AICD-mediated luciferase activity induced by TrkB SHC might be mediated by binding of SHC adaptor proteins. Binding of adaptor proteins to TrkB and possibly to APP, might decrease the endocytosis of APP decreasing its $\beta$-secretase cleavage [44]. The luciferase assay described here has been found to be particularly sensitive in detecting decreased $\beta$-secretase processing [45] and that can be the cause of the decrease in luciferase activity that we observe, at least with cotransfection of the TrkB SHC isoform.

Our data demonstrates differential effects of the TrkB isoforms on AICD-mediated transcription showing that TrkB SHC behaves differently from both TrkB FL and TrkB T. It has been previously demonstrated that BDNF application does not improve the cognitive function in a trisomy 21 mouse model because $\operatorname{TrkB} \mathrm{T}$ is upregulated [43, 46]. Therefore, a better understanding of the individual TrkB isoforms and their signaling role will improve the therapeutic potential of BDNF or BDNF agonists.

Experimentally, we found that the detected protein levels of TrkB FL were much lower than TrkB T and TrkB SHC levels. We can exclude effects due to plasmid copy number in the cells since we used equimolar amounts of plasmid DNA that account for differences in plasmid size. We can also exclude differences in transcription levels due to plasmid promoters since the TrkB SHC vector was generated by mutagenesis of the TrkB FL vector. The difference in expression levels of the TrkB isoforms is highly reproducible suggesting that there 
might be a tight regulation of TrkB FL expression levels. TrkB FL is stored in intracellular vesicles that rapidly fuse to the cell membrane upon BDNF stimulation of the cells [37]. This causes a fast BDNF-mediated phosphorylation of the receptor and initiates intracellular signaling [47]. After this spike of activity TrkB/BDNF complexes are rapidly endocytosed and degraded [48]. High TrkB FL expression levels increase malignancy in neuroblastomas reinforcing the idea that regulatory mechanisms of $\operatorname{TrkB}$ expression and signaling are necessary to maintain homeostasis $[49,50]$. TrkB FL expression is also decreased by chronic BDNF stimulation of $\mathrm{H} 4$ neuroblastoma cells while TrkB $\mathrm{T}$ levels remain constant [51]. We therefore hypothesize that in our model system, TrkB FL levels are controlled by mechanisms that cannot be overcome by TrkB FL overexpression and that BDNF expressed by the cell line might be one of the causes of this downregulation.

To determine which TrkB functional domain and signaling pathway was mediating the TrkB mediate effects, we overexpressed the mutant TrkB isoforms and monitored AICD-mediated luciferase activity. The observed TrkB FLmediated increase in luciferase activity was suppressed by either inactivating the tyrosine kinase activity (K571M) or mutating the PLC- $\gamma$-binding site (Y816F). We hypothesize that the PLC- $\gamma$ effect is due to lack of PLC- $\gamma$ activation which produces DAG (Diacyl Glycerol), an activator of PKC, a protein that mediates ADAM10 activation [52]. The fact that there is a difference between the TrkB K571M mutant and the TrkB Y816F PLC- $\gamma$-binding site mutant suggests both of these functional domains and their associated pathways can regulate APP metabolism.

The SHC-binding site on the TrkB FL receptor did not seem to be involved in mediating increased AICDmediated luciferase activity since the TrkB Y515F mutant did not differ from the TrkB FL isoform in increasing AICD-mediated luciferase activity. Also the AICD-mediated luciferase signal in cells transfected with the double mutant TrkB K571M/Y515F did not differ from the cells transfected with the $\operatorname{TrKB} \mathrm{K} 571 \mathrm{M}$ mutant suggesting that there is no additive effect in eliminating both signaling pathways. This does not completely exclude a role for the SHCbinding domain. In fact, the binding of SHC might occur more efficiently when the site is phosphorylated so that when phosphorylation is prevented the small change in luciferase signal is not detectable in our experimental system. Supporting this hypothesis is a nonsignificant decrease of AICD-mediated activity caused by the TrkB Y515F mutant compared to TrkB FL.

We observe a significant effect when the TrkB SHC isoform is mutated to eliminate SHC-binding. This mutant induces the same luciferase activity signal of the TrkB T. This finding suggests that binding of SHC to the TrkB SHC isoform might mediate signaling pathways independently of phosphorylation. Importantly, we demonstrate that there is a difference in signal transduction between the two truncated TrkB isoforms and that they act on APP-mediated transcription. Moreover, we identify the SHC-binding domain as responsible for the difference in signaling mechanism between TrkB T and SHC. Mutation of the binding site for
SHC adaptor proteins on the truncated TrkB SHC isoform increases AICD-mediated luciferase signal, while the same SHC site on the TrkB FL is not responsible for the observed changes in luciferase activity. This contrasting result suggests that interaction between the same proteins and specific TrkB isoforms mediates different signaling pathways.

The cell line used, SH-SY5Y, expresses basal levels of TrkB receptors and BDNF [31], the endogenously expressed BDNF can promote dimerization and activation of the overexpressed receptors. Also, BDNF independent activation of TrkB FL receptors has been previously demonstrated [53] and we hypothesize that both BDNF independent and dependent activation coexist in our experimental system. Endogenous TrkB receptors might also be upregulated or downregulated in response to exogenous TrkB expression.

To assess the effect of BDNF-dependent activation we added exogenous BDNF to the transfected cells. BDNF is hypothesized to activate the receptors by mediating their dimerization [12]. In our experimental system BDNF treatment did not significantly alter the effects of the TrkB isoform overexpression on APP FL levels. It has been observed before that TrkB FL overexpression can cause receptor autoactivation [53]. Our data suggests that a similar mechanism occurs in our experimental system. The close proximity of the overexpressed receptors on the membrane probably allows dimerization and activation of the receptors independently from BDNF so that even when BDNF is added to the system any additional effect on TrkB activation is not detectable.

We mentioned above that SH-SY5Y cells express basal levels of the TrkB receptors. To investigate the role of $\operatorname{TrkB}$ isoforms interaction on TrkB FL-mediated signaling, we coexpressed exogenous TrkB FL with truncated isoforms and mutated variants. Cotransfection of the TrkB FL with the truncated $\mathrm{T}$ and $\mathrm{SHC}$ isoforms or the tyrosine kinase inactive mutant abrogated the increase in APP FL levels induced by TrkB FL. Interestingly TrkB FL/SHC cotransfection had higher APP FL levels than TrkB FL/T cotransfection. This points to a possible difference between the two TrkB truncated isoforms in the regulation of the TrkB FL catalytic receptor. The fact that in the cotransfection experiments TrkB FL/SHC showed increased APP FL levels compared to TrkB FL/T also suggests that SHC-binding to this isoform might occur more efficiently when TrkB FL and TrkB SHC interact, maybe causing phosphorylation on the SHCbinding site. Cotransfection of TrkB FL/Y515F had similar effects on APP FL to TrkB FL/FL but was less effective in inducing an increase in APP FL levels. TrkB FL/Y816F cotransfection was indistinguishable from TrkB FL single transfection suggesting that PLC- $\gamma$ signaling is not involved in determining APP FL levels. BDNF treatment of the cotransfected cells seemed to accentuate the effect of TrkB FL on APP FL levels. For example, it increased APP FL in cells cotransfected with TrkB FL/T but not in cells cotransfected with TrkB FL/K571M. On the contrary, TrkB FL/Y515F cotransfection seemed to cause lower APP FL levels when BDNF was applied. It is intriguing to think that when all TrkB isoforms are expressed in the cells, as should be the case in our model, BDNF promotes homodimerization versus 
heterodimerization. The issue of preferential homo- versus heterointeraction of TrkB isoforms has not been investigated so far and it would be important to address.

\section{Conclusions}

This work demonstrates that truncated TrkB isoforms affect APP processing and transcriptional signaling differently than full-length TrkB. Not only do the truncated isoforms have a different effects when transfected alone, they were also able to modify the TrkB FL effects when co-transected with it. These findings point to the possible roles of the TrkB isoforms in the pathogenesis of $\mathrm{AD}$. In fact all the isoforms are present on neurons and other cell types of the CNS. The proportion of $\operatorname{TrkB}$ FL to $\operatorname{TrkB} \mathrm{T}$ and $\operatorname{TrkB} \mathrm{SHC}$ is then important in determining the effect on $\operatorname{TrkB}$ signal transduction and APP metabolism. Since all the isoforms bind BDNF in the extracellular domain, a therapeutic approach that uses BDNF biomimetic drugs might not be as effective as if only TrkB FL was expressed. In fact, expression of truncated isoforms could scavenge the drugs, decrease the benefit of engaging TrkB FL triggered pathways, and also inhibit the TrkB FL effects. Depending on the relative amounts of the TrkB receptors on the cells, BDNF-mimetic drugs could cause an overall worsening of the conditions [46] by, for example, increasing the inflammatory response. It will be important in the future to dissect the contributions of the TrkB isoforms to BDNF-dependent and -independent signaling pathways in the context of $\mathrm{AD}$ to better understand which isoforms and pathways are beneficial and which ones are detrimental.

\section{References}

[1] J. Wancata, M. Musalek, R. Alexandrowicz, and M. Krautgartner, "Number of dementia sufferers in Europe between the years 2000 and 2050," European Psychiatry, vol. 18, no. 6, pp. 306-313, 2003.

[2] Alzheimer's, "2010 Alzheimer's disease facts and figures," Alzheimer's and Dementia, vol. 6, no. 2, pp. 158-194, 2010.

[3] M. Gatz, C. A. Reynolds, L. Fratiglioni et al., "Role of genes and environments for explaining Alzheimer disease," Archives of General Psychiatry, vol. 63, no. 2, pp. 168-174, 2006.

[4] D. G. Munoz and H. Feldman, "Causes of Alzheimer's disease," Canadian Medical Association Journal, vol. 162, no. 1, pp. 6572, 2000.

[5] N. Scarmeas, J. A. Luchsinger, N. Schupf et al., "Physical activity, diet, and risk of Alzheimer disease," Journal of the American Medical Association, vol. 302, no. 6, pp. 627-637, 2009.

[6] M. Khvotchev and T. C. Südhof, "Proteolytic processing of amyloid- $\beta$ precursor protein by secretases does not require cell surface transport," The Journal of Biological Chemistry, vol. 279, no. 45, pp. 47101-47108, 2004.

[7] B. N. Ramesh, T. S. S. Rao, A. Prakasam, K. Sambamurti, and K. S. J. Rao, "Neuronutrition and Alzheimer's disease," Journal of Alzheimer's Disease, vol. 19, no. 4, pp. 1123-1139, 2010.

[8] D. J. Selkoe, "Soluble oligomers of the amyloid $\beta$-protein impair synaptic plasticity and behavior," Behavioural Brain Research, vol. 192, no. 1, pp. 106-113, 2008.

[9] D. Kim and L. H. Tsai, "Bridging Physiology and Pathology in AD," Cell, vol. 137, no. 6, pp. 997-1000, 2009.
[10] E. Marcello, R. Epis, and M. Di Luca, "Amyloid flirting with synaptic failure: towards a comprehensive view of Alzheimer's disease pathogenesis," European Journal of Pharmacology, vol. 585, no. 1, pp. 109-118, 2008.

[11] L. Minichiello, M. Korte, D. Wolfer et al., "Essential role for TrkB receptors in hippocampus-mediated learning," Neuron, vol. 24, no. 2, pp. 401-414, 1999.

[12] L. F. Reichardt, "Neurotrophin-regulated signalling pathways," Philosophical Transactions of the Royal Society B, vol. 361, no. 1473, pp. 1545-1564, 2006.

[13] S. D. Ginsberg, S. Che, J. Wuu, S. E. Counts, and E. J. Mufson, "Down regulation of trk but not p75 gene expression in single cholinergic basal forebrain neurons mark the progression of Alzheimer's disease," Journal of Neurochemistry, vol. 97, no. 2, pp. 475-487, 2006.

[14] S. Holback, L. Adlerz, and K. Iverfeldt, "Increased processing of APLP2 and APP with concomitant formation of APP intracellular domains in BDNF and retinoic acid-differentiated human neuroblastoma cells," Journal of Neurochemistry, vol. 95, no. 4, pp. 1059-1068, 2005.

[15] Y. Ruiz-León and A. Pascual, "Brain-derived neurotrophic factor stimulates $\beta$-amyloid gene promoter activity by a rasdependent/AP-1-independent mechanism in SH-SY5Y neuroblastoma cells," Journal of Neurochemistry, vol. 79, no. 2, pp. 278-285, 2001.

[16] Y. Ruiz-León and A. Pascual, "Induction of tyrosine kinase receptor B by retinoic acid allows brain-derived neurotrophic factor-induced amyloid precursor protein gene expression in human SH-SY5Y neuroblastoma cells," Neuroscience, vol. 120, no. 4, pp. 1019-1026, 2003.

[17] Y. Ruiz-León and A. Pascual, "Regulation of $\beta$-amyloid precursor protein expression by brain-derived neurotrophic factor involves activation of both the Ras and phosphatidylinositide 3-kinase signalling pathways," Journal of Neurochemistry, vol. 88, no. 4, pp. 1010-1018, 2004.

[18] L. Tong, R. Balazs, P. L. Thornton, and C. W. Cotman, “ $\beta$ amyloid peptide at sublethal concentrations downregulates brain-derived neurotrophic factor functions in cultured cortical neurons," Journal of Neuroscience, vol. 24, no. 30, pp. 67996809, 2004.

[19] G. Olivieri, U. Otten, F. Meier et al., " $\beta$-amyloid modulates tyrosine kinase B receptor expression in SHSY5Y neuroblastoma cells: influence of the antioxidant melatonin," Neuroscience, vol. 120, no. 3, pp. 659-665, 2003.

[20] W. W. Poon, M. Blurton-Jones, C. H. Tu et al., " $\beta$-Amyloid impairs axonal BDNF retrograde trafficking," Neurobiology of Aging. In press.

[21] Y. Zeng, D. Zhao, and C.-W. Xie, "Neurotrophins enhance CaMKII activity and rescue amyloid- $\beta$-induced deficits in hippocampal synaptic plasticity," Journal of Alzheimer's Disease, vol. 21, no. 3, pp. 823-831, 2010.

[22] M. M. Carrasquillo, O. Belbin, T. A. Hunter et al., "Replication of CLU, CR1, and PICALM associations with Alzheimer disease," Archives of Neurology, vol. 67, no. 8, pp. 961-964, 2010.

[23] K. Michaelsen, M. Zagrebelsky, J. Berndt-Huch et al., "Neurotrophin receptors TrkB.T1 and p75(NTR) cooperate in modulating both functional and structural plasticity in mature hippocampal neurons," European Journal of Neuroscience, vol. 32, no. 11, pp. 1854-1865, 2010.

[24] M. L. Hamshere, P. A. Holmans, D. Avramopoulos et al., "Genome-wide linkage analysis of 723 affected relative pairs with late-onset Alzheimer's disease," Human Molecular Genetics, vol. 16, no. 22, pp. 2703-2712, 2007. 
[25] R. T. Perry, H. Wiener, L. E. Harrell et al., "Follow-up mapping supports the evidence for linkage in the candidate region at $9 \mathrm{q} 22$ in the NIMH Alzheimer's disease genetics initiative cohort," American Journal of Medical Genetics, Part B, vol. 144, no. 2, pp. 220-227, 2007.

[26] Z. Chen, M. S. Simmons, R. T. Perry, H. W. Wiener, L. E. Harrell, and R. C. P. Go, "Genetic association of neurotrophic tyrosine kinase receptor type 2 (NTRK2) with Alzheimer's disease," American Journal of Medical Genetics, Part B, vol. 147, no. 3, pp. 363-369, 2008.

[27] A. Cozza, E. Melissari, P. Iacopetti et al., "SNPs in neurotrophin system genes and Alzheimer's disease in an Italian population," Journal of Alzheimer's Disease, vol. 15, no. 1, pp. 61-70, 2008.

[28] S. Vepsäläinen, E. Castren, S. Helisalmi et al., "Genetic analysis of BDNF and TrkB gene polymorphisms in Alzheimer's disease," Journal of Neurology, vol. 252, no. 4, pp. 423-428, 2005.

[29] H. Li, S. Wetten, L. Li et al., "Candidate single-nucleotide polymorphisms from a genomewide association study of Alzheimer disease," Archives of Neurology, vol. 65, no. 1, pp. 45-53, 2008.

[30] E. M. Reiman, J. A. Webster, A. J. Myers et al., "GAB2 alleles modify Alzheimer's risk in APOE epsilon4 carriers," Neuron, vol. 54, no. 5, pp. 713-720, 2007.

[31] P. Stoilov, E. Castren, and S. Stamm, "Analysis of the human TrkB gene genomic organization reveals novel TrkB isoforms, unusual gene length, and splicing mechanism," Biochemical and Biophysical Research Communications, vol. 290, no. 3, pp. 1054-1065, 2002.

[32] D. S. Middlemas, R. A. Lindberg, and T. Hunter, "trkB, a neural receptor protein-tyrosine kinase: evidence for a fulllength and two truncated receptors," Molecular and Cellular Biology, vol. 11, no. 1, pp. 143-153, 1991.

[33] J. K. Atwal, B. Massie, F. D. Miller, and D. R. Kaplan, "The TrkB-Shc site signals neuronal survival and local axon growth via MEK and PI3-Kinase," Neuron, vol. 27, no. 2, pp. 265-277, 2000.

[34] K. Luberg, J. Wong, C. S. Weickert, and T. Timmusk, "Human TrkB gene: novel alternative transcripts, protein isoforms and expression pattern in the prefrontal cerebral cortex during postnatal development," Journal of Neurochemistry, vol. 113, no. 4, pp. 952-964, 2010.

[35] C. Zhang, P. J. Khandelwal, R. Chakraborty et al., "An AICD-based functional screen to identify APP metabolism regulators," Molecular Neurodegeneration, vol. 2, no. 1, article 15, 2007.

[36] A. Haapasalo, T. Saarelainen, M. Moshnyakov et al., "Expression of the naturally occurring truncated trkB neurotrophin receptor induces outgrowth of filopodia and processes in neuroblastoma cells," Oncogene, vol. 18, no. 6, pp. 1285-1296, 1999.

[37] A. Haapasalo, I. Sipola, K. Larsson et al., "Regulation of TRKB surface expression by brain-derived neurotrophic factor and truncated TRKB isoforms," The Journal of Biological Chemistry, vol. 277, no. 45, pp. 43160-43167, 2002.

[38] L. Minichiello, "TrkB signalling pathways in LTP and learning," Nature Reviews Neuroscience, vol. 10, no. 12, pp. 850-860, 2009.

[39] D. Blacker, L. Bertram, A. J. Saunders et al., "Results of a highresolution genome screen of 437 Alzheimer's Disease families," Human Molecular Genetics, vol. 12, no. 1, pp. 23-32, 2003.

[40] F. Kamenetz, T. Tomita, H. Hsieh et al., "APP Processing and Synaptic Function,” Neuron, vol. 37, no. 6, pp. 925-937, 2003.
[41] X. Cao and T. C. Südhof, "Dissection of amyloid- $\beta$ precursor protein-dependent transcriptional transactivation," The Journal of Biological Chemistry, vol. 279, no. 23, pp. 24601-24611, 2004.

[42] A. Haapasalo, E. Koponen, E. Hoppe, G. Wong, and E. Castrén, "Truncated trkB.T1 is dominant negative inhibitor of trkB.TK+-mediated cell survival," Biochemical and Biophysical Research Communications, vol. 280, no. 5, pp. 1352-1358, 2001.

[43] S. G. Dorsey, C. L. Renn, L. Carim-Todd et al., "In vivo restoration of physiological levels of truncated TrkB.T1 receptor rescues neuronal cell death in a trisomic mouse model," Neuron, vol. 51, no. 1, pp. 21-28, 2006.

[44] S. F. Lichtenthaler, "Ectodomain shedding of the amyloid precursor protein: cellular control mechanisms and novel modifiers," Neurodegenerative Diseases, vol. 3, no. 4-5, pp. 262-269, 2006.

[45] S. E. Hoey, R. J. Williams, and M. S. Perkinton, "Synaptic NMDA receptor activation stimulates $\alpha$-secretase amyloid precursor protein processing and inhibits amyloid- $\beta$ production," Journal of Neuroscience, vol. 29, no. 14, pp. 4442-4460, 2009.

[46] S. G. Dorsey, L. L. Bambrick, R. J. Balice-Gordon, and B. K. Krueger, "Failure of brain-derived neurotrophic factordependent neuron survival in mouse trisomy 16," Journal of Neuroscience, vol. 22, no. 7, pp. 2571-2578, 2002.

[47] J. Du, L. Feng, E. Zaitsev, H. S. Je, X. W. Liu, and B. $\mathrm{Lu}$, "Regulation of TrkB receptor tyrosine kinase and its internalization by neuronal activity and $\mathrm{Ca}^{2+}$ influx," Journal of Cell Biology, vol. 163, no. 2, pp. 385-395, 2003.

[48] M. T. Sommerfeld, R. Schweigreiter, Y. A. Barde, and E. Hoppe, "Down-regulation of the neurotrophin receptor TrkB following ligand binding. Evidence for an involvement of the proteasome and differential regulation of TrkA and TrkB," The Journal of Biological Chemistry, vol. 275, no. 12, pp. 89828990, 2000.

[49] S. Douma, T. Van Laar, J. Zevenhoven, R. Meuwissen, E. Van Garderen, and D. S. Peeper, "Suppression of anoikis and induction of metastasis by the neurotrophic receptor TrkB," Nature, vol. 430, no. 7003, pp. 1034-1040, 2004.

[50] C. J. Thiele, Z. Li, and A. E. McKee, "On Trk-the TrkB signal transduction pathway is an increasingly important target in cancer biology," Clinical Cancer Research, vol. 15, no. 19, pp. 5962-5967, 2009.

[51] M. Rohe, M. Synowitz, R. Glass, S. M. Paul, A. Nykjaer, and T. E. Willnow, "Brain-derived neurotrophic factor reduces amyloidogenic processing through control of SORLA gene expression," Journal of Neuroscience, vol. 29, no. 49, pp. 1547215478, 2009.

[52] S. Lammich, E. Kojro, R. Postina et al., "Constitutive and regulated $\alpha$-secretase cleavage of Alzheimer's amyloid precursor protein by a disintegrin metalloprotease," Proceedings of the National Academy of Sciences of the United States of America, vol. 96, no. 7, pp. 3922-3927, 1999.

[53] L. C. Schecterson, M. P. Hudson, M. Ko et al., "Trk activation in the secretory pathway promotes Golgi fragmentation," Molecular and Cellular Neuroscience, vol. 43, no. 4, pp. 403413, 2010. 


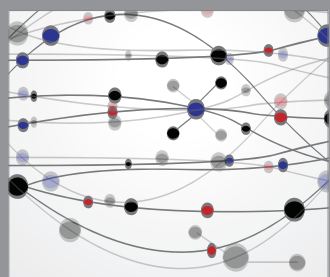

The Scientific World Journal
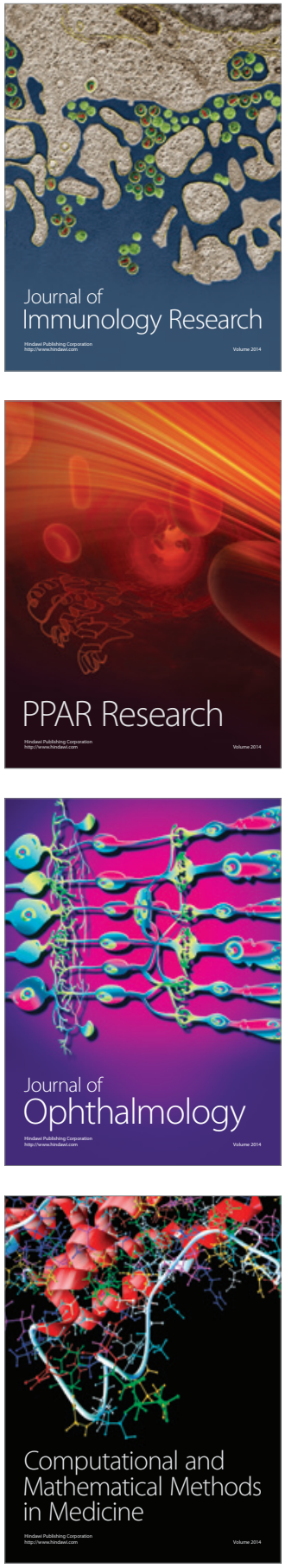

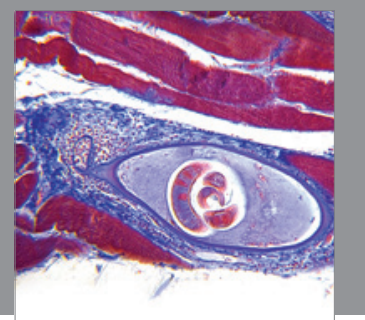

Gastroenterology

Research and Practice
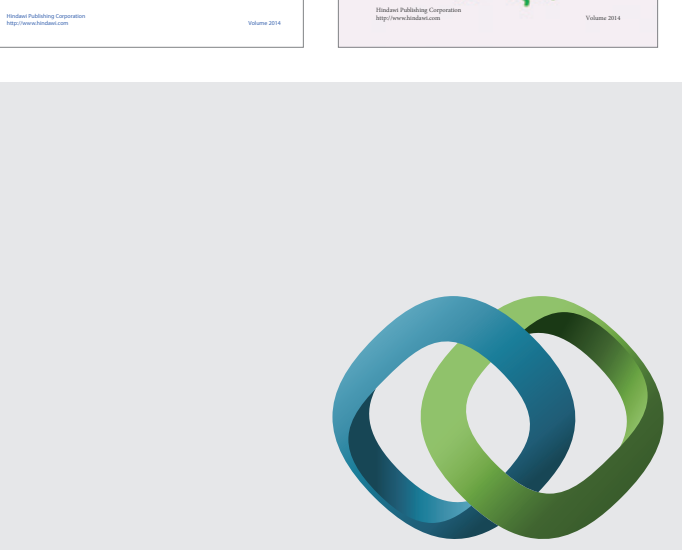

\section{Hindawi}

Submit your manuscripts at

http://www.hindawi.com
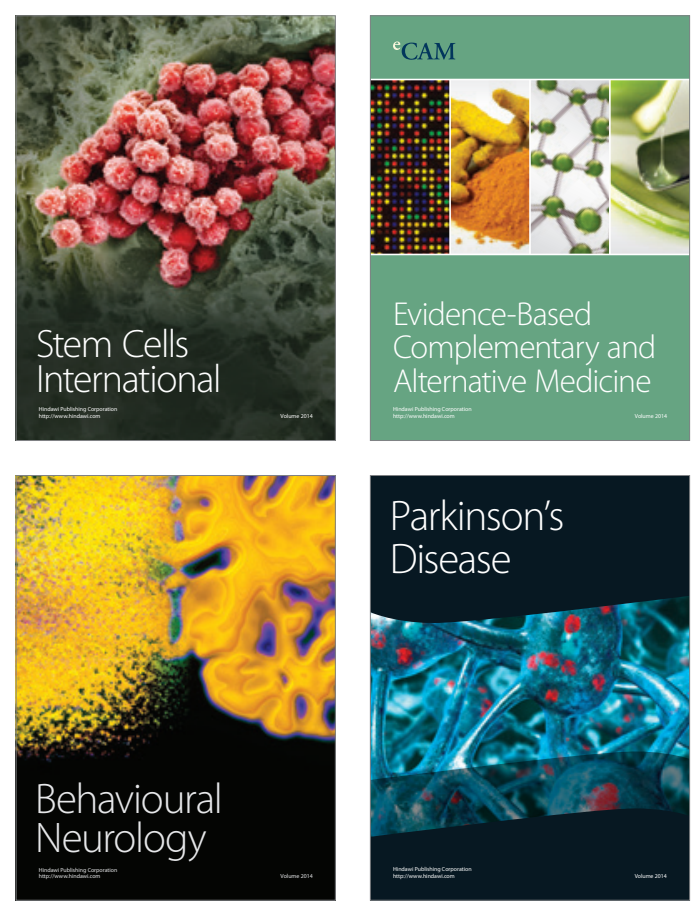

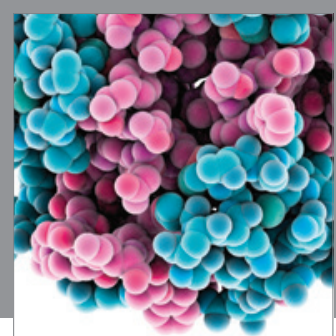

Journal of
Diabetes Research

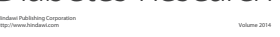

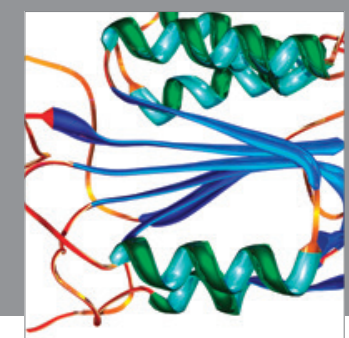

Disease Markers
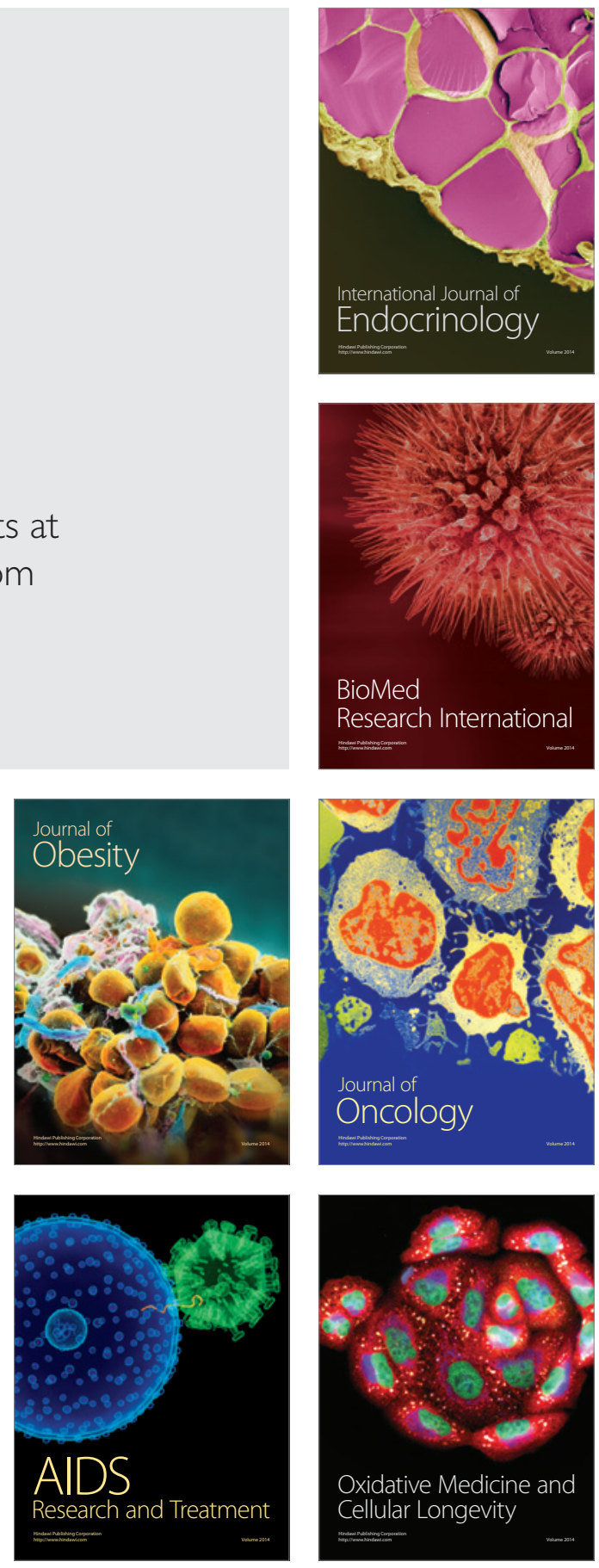